\title{
ESTUDO DAS TENSÕES RESIDUAIS E DISTORÇÕES DE PEÇAS PRODUZIDAS POR IMPRESSÃO FDM UTILIZANDO O MÉTODO DOS ELEMENTOS
}

\section{STUDY OF RESIDUAL STRESS AND DISTORTIONS OF PARTS PRODUCED BY FDM PRINTING USING THE FINITE ELEMENT METHOD}

\author{
A. T. MALAVOLTA ${ }^{1, *}$, A. G. MÜLLER ${ }^{1}$
}

${ }^{1}$ Universidade Federal de São Carlos, Departamento de Engenharia Mecânica, São Carlos, SP, Brasil

${ }^{*}$ Corresponding author. Federal University of São Carlos, Department of Mechanical Engineering, São Carlos, São Paulo, Brazil, Phone: +551633066418 e-mail addressl: malavolta@ufscar.br (A.T. Malavolta).

\begin{tabular}{l} 
A R T I C L E I N F O \\
\hline Article history: \\
Received 2020-09-26 \\
Accepted 2020-12-12 \\
Available online 2020-12-12 \\
p a l a vra - c h a ve \\
Manufatura aditiva \\
Modelagem por fusão e deposição \\
Método dos elementos finitos \\
ke y wo r $d s$ \\
Additive manufacturing \\
Fused deposition modeling \\
Finite element method
\end{tabular}

\begin{abstract}
A B S T R A C T
In FDM (Fused Deposition Modeling) additive manufacturing process, filaments of polymeric materials are fused in an extruder nozzle and deposited in layers to produce a piece with predetermined geometry. During the extrusion process, the polymeric material reaches high temperatures, changing the phase and cooling rapidly after the deposition of the layers, which occurs repeatedly until the end of the process. Thus, it is common for thermal gradients to cause residual stresses and distortions in the parts produced. In this context, this work uses the finite element method to perform thermo-mechanical analysis and to predict the distortions and residual stresses in a part produced by the FDM process.

R E S U M O

No processo de manufatura aditiva FDM (Modelagem por Fusão e Deposição) filamentos de materiais poliméricos são fundidos em um bocal extrusor e depositados em camadas para produzir uma peça com geometria pré-determinada através de um modelo. Durante o processo de extrusão, o material polimérico atinge elevadas temperaturas, mudando de fase e resfriando-se rapidamente após a deposição das camadas, o que ocorre repetidamente até o final do processo. Desta forma, é comum que os gradientes térmicos acarretem tensões residuais e distorções nas peças produzidas. Neste contexto, este trabalho utiliza o método dos elementos finitos para realizar análises termo-mecânicas e prever as distorções e tensões residuais em uma peça produzida pelo processo FDM.
\end{abstract}




\section{N O M E N C L A T UR E}

$\sigma_{\mathrm{X}}$ : Componente de tensão direção x [MPa]

$\sigma_{\mathrm{Y}}$ : Componente de tensão direção y [MPa]

$\sigma_{\mathrm{Z}}$ : Componente de tensão direção z [MPa]

$\tau_{\mathrm{XY}}$ : Componente de tensão de cisalhamento xy [MPa]

$\tau_{\mathrm{YZ}}$ : Componente de tensão de cisalhamento yz [MPa]

$\tau_{\mathrm{ZX}}$ : Componente de tensão de cisalhamento zx [MPa]

$\mathrm{u}_{\mathrm{z}}^{\mathrm{m}}$ : Deslocamentos do modelo $[\mu \mathrm{m}]$

$\mathrm{u}_{\mathrm{z}}{ }^{\mathrm{e}}$ : Deslocamentos experimentais $[\mu \mathrm{m}]$

\section{INTRODUÇÃO}

$\mathrm{O}$ processo de manufatura aditiva FDM (Fused Deposition Modeling) consiste na extrusão de filamentos poliméricos, usualmente PLA (ácido polilático) ou ABS (acronitrila butadieno estireno) em diâmetros de 1,75 mm ou 3 $\mathrm{mm}$. Os filamentos são empurrados por um rolo alimentador para dentro de uma câmara de aquecimento onde são liquefeitos, gerando uma pressão de extrusão. O material liquefeito é então depositado pelo bocal, cujo diâmetro varia de $0,1 \mathrm{~mm}$ a $1 \mathrm{~mm}$, sobre uma plataforma aquecida, camada sobre camada, até atingir a forma final do componente. A Figura 1 ilustra o funcionamento desse processo.

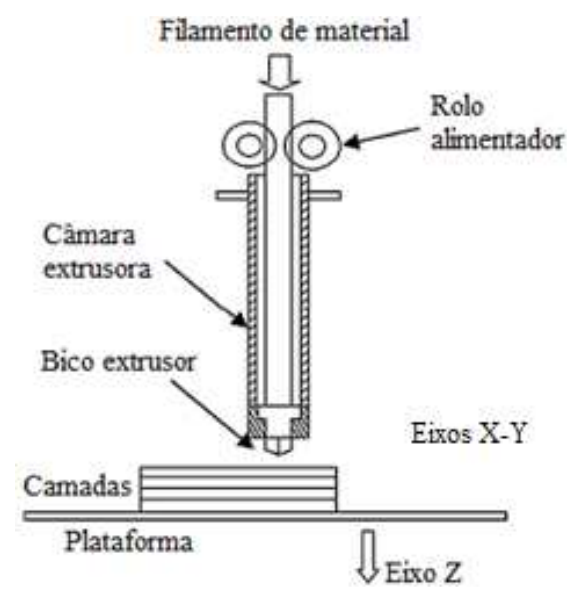

Figura 1 - Esquema de funcionamento do processo FDM. Fonte: Adaptado de Gibson et al. (2015).

Apesar do processo FDM ser uma técnica bastante utilizada e difundida, as condições de operação muitas vezes afetam a configuração final da peça produzida, tal que as especificações de projeto podem não ser atendidas. Durante o processo de extrusão, o material polimérico atinge elevadas temperaturas, mudando de fase e em seguida resfriando-se por condução, convecção e radiação antes que a próxima camada de material fundido seja depositada no topo, o que ocorre repetidamente até o final do processo. Este gradiente térmico é responsável pelas tensões residuais acumuladas que causam empenamento, distorção e afetam a geometria do produto, bem como o desempenho em serviço.
Para minimizar estes problemas, diversos pesquisadores desenvolvem trabalhos para investigar as distorções geradas durante o processo de deposição FDM. Armillotta et al. (2018), por exemplo, realizaram testes experimentais e desenvolveram modelos teóricos a fim de caracterizar o empenamento de peças produzidas em ABS pelo processo FDM. A Figura 2 ilustra as características do empenamento resultante do processo FDM devido aos efeitos térmicos. As variáveis consideradas pelo estudo dos autores foram as dimensões da peça e a espessura das camadas depositadas. Os desvios geométricos de peças fabricadas com diferentes combinações dessas variáveis foram mensurados e analisados estatisticamente a fim de identificar os fatores de influência e estimar seus efeitos individuais na distorção da peça produzida.

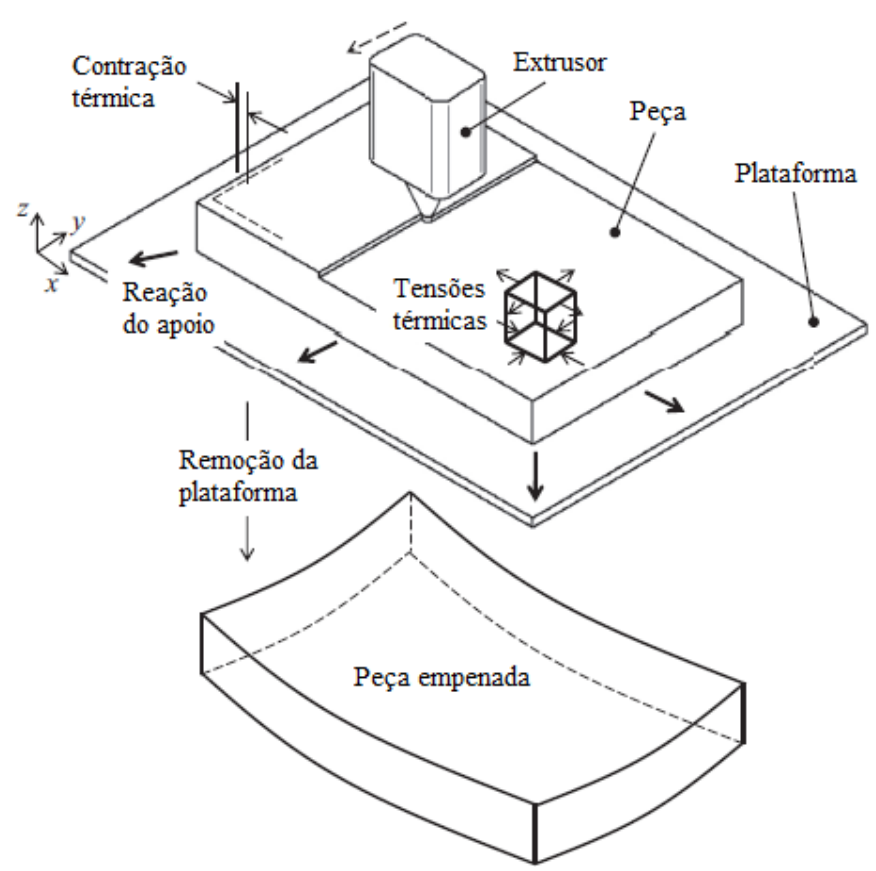

Figura 2 - Empenamento no processo FDM. Fonte: Adaptado de Armillotta et al. (2018).

Li et al. (2018) apresentam uma proposta de investigação quantitativa da distorção de uma peça de PLA produzida por FDM. Nessa pesquisa os parâmetros considerados para análise foram a espessura das camadas e a velocidade de deposição. Os resultados obtidos indicam que a espessura de camada é dominante na determinação da distorção da peça final em comparação com a velocidade de deposição.

Zhang e Chou (2006) apresentaram um modelo de elementos finitos utilizando o recurso de ativação e desativação de elementos no software ANSYS através de um acoplamento termomecânico. A sequência de modelagem consiste em realizar uma simulação térmica e aplicar o gradiente térmico obtido na simulação mecânica a fim de obter os deslocamentos e tensões residuais. A utilização do recurso de ativação e desativação consiste em desativar todos os elementos após a definição da malha; quando um elemento é ativado, simulando a deposição do material, a análise térmica transiente é iniciada considerando a distribuição inicial de temperaturas. $\mathrm{O}$ cálculo dessa etapa continua durante o intervalo de deposição do elemento, em seguida o próximo elemento é ativado e a análise 
é iniciada novamente considerando as temperaturas atualizadas. Uma vez que todos os elementos foram ativados, o cálculo continua até que o modelo atinja o equilíbrio térmico. O resultado final da distribuição de temperaturas é utilizado como carregamento para a análise mecânica, que calcula o deslocamento em cada nó, bem como a tensão residual.

Yang e Zhang (2018) também propõe a simulação numérica dos campos de temperatura e tensão do processo de deposição de material fundido. A sequência de modelagem é análoga à utilizada por Zhang e Chou (2006) e as hipóteses assumidas para a análise foram que o filamento polimérico possui forma cilíndrica, a temperatura inicial de cada elemento é igual à temperatura de saída do bocal, o material depositado realiza transferência de calor com o ar, o material segue a teoria plástica de deformação e as deformações estão relacionadas com o gradiente térmico.

Outro estudo similar é o de Zhang e Chou (2008), que analisou os efeitos dos parâmetros do processo FDM, tais como largura do caminho de deposição, espessura de camada, velocidade de deposição, através de um modelo tridimensional de elementos finitos. $\mathrm{O}$ resultado das simulações mostra que as distorções são relacionadas à tensão acumulada durante a deposição do material e, nesse estudo, a velocidade de deposição foi o fator mais significante no que se refere à distorção final da peça, seguida pela espessura de camada.

El Moumen et al. (2019) criaram um modelo que considera as propriedades físicas dependentes da temperatura, tais como densidade, condutividade térmica, coeficiente de expansão térmica e módulo de elasticidade. A peça simulada consiste em um corpo de prova produzido em poliamida e a metodologia inclui as fases de aquecimento, solidificação e resfriamento. Assim como nos estudos citados anteriormente, realizou-se primeiramente uma simulação térmica, com a temperatura variando ao longo do tempo de impressão e, em seguida, o campo de temperaturas foi utilizado para calcular as tensões residuais através de uma simulação termoelástica. Para simular o processo de impressão FDM foi utilizada a função de ativação/desativação de elementos no software Digimat.

Cattenone et al. (2019) realizaram um estudo detalhado de diversos parâmetros sobre o comportamento mecânico de componentes impressos em ABS utilizando o software ABAQUS com o recurso de ativação e desativação de elementos. A influência dos parâmetros de simulação, tais como tamanho da malha e tempo da simulação térmica transiente foram analisados em detalhes. Por fim, os resultados da simulação foram comparados com testes experimentais para validação do modelo.

Neste contexto, o objetivo principal deste trabalho é a investigação das distorções e tensões residuais em peças produzidas pela técnica de manufatura aditiva FDM. Modelos computacionais, empregando o método dos elementos finitos, foram elaborados para obter uma previsão das distorções e dos campos de tensão nas peças utilizando uma abordagem diferente da identificada na revisão bibliográfica, ou seja, sem a utilização do recurso de ativação e desativação dos elementos durante a simulação.

\section{MATERIAIS E MÉTODOS}

As simulações de previsão das distorções utilizando o método dos elementos finitos foram realizadas com o software NX Siemens 12, em um computador com processador Intel Core i7-7500, CPU 2.7 GHz e com 8GB de memória RAM.

Primeiramente, foram realizadas simulações térmicas transientes de um único fio sendo depositado a fim de prever o padrão das tensões residuais atuantes. Para fins de comparação, foram definidos cinco comprimentos de fios, sendo eles: $15 \mathrm{~mm}$, $20 \mathrm{~mm}, 40 \mathrm{~mm}, 80 \mathrm{~mm}$ e $160 \mathrm{~mm}$. A seção transversal do fio foi aproximada como um quadrado de dimensões $1 \mathrm{~mm}$ x $1 \mathrm{~mm}$. Para cada um dos cinco fios foi feito um modelo em CAD e, em seguida, definiu-se uma malha para a realização das simulações utilizando elementos hexaédricos de 8 nós. As propriedades térmicas e mecânicas do ABS foram adotadas conforme Cattenone et al. (2019). Como condições de contorno, definiuse a temperatura ambiente de $45^{\circ} \mathrm{C}$, simulando um ambiente com temperatura controlada e, foram definidas convecções das superfícies laterais e superior do fio para o ambiente e de sua superfície inferior para a mesa, a $90^{\circ} \mathrm{C}$. Os coeficientes convectivos para as superfícies laterais e superior foram definidos como $10 \mathrm{~W} /\left(\mathrm{m}^{2} .{ }^{\circ} \mathrm{C}\right)$ de acordo com valores recomendados considerando uma convecção natural, enquanto que para a superfície inferior, adotou-se $400 \mathrm{~W} /\left(\mathrm{m}^{2}{ }^{\circ} \mathrm{C}\right)$, valor obtido após uma sequência de testes para simular o contato direto entre o filamento e a mesa aquecida.

A simulação térmica transiente foi realizada considerando uma abordagem diferente dos estudos previamente apresentados para simular a deposição do material. Visando reduzir o tempo de simulação, optou-se por não utilizar o recurso de ativação e desativação dos elementos. Dessa forma, a simulação da deposição do ABS foi realizada aplicando uma taxa de geração de calor que se move ao longo do comprimento do fio com uma velocidade de $16 \mathrm{~mm} / \mathrm{s}$ e aquece os elementos até a temperatura de extrusão $\left(240^{\circ} \mathrm{C}\right)$. Tal aproximação é capaz de representar a deposição do material, pois ainda que os elementos estejam ativos na simulação, a sua temperatura só é alterada quando o fluxo de calor passa por eles, simulando o movimento do bico extrusor. Após o término da simulação térmica o mapa de temperaturas do fio é exportado, considerando o instante em que é atingida a temperatura de transição vítrea $\left(95^{\circ} \mathrm{C}\right)$, pois, acima dessa temperatura a rigidez do ABS é praticamente nula.

Em seguida, o mapa de temperaturas obtido é utilizado como carregamento em uma nova simulação termoelástica do fio para determinar-se as componentes de tensão resultantes do gradiente térmico. Neste caso, como condição de contorno, o deslocamento de toda a base do fio é restringido. A partir desta simulação termoelástica, pode-se avaliar o padrão das componentes de tensão atuantes, normais e de cisalhamento $\left(\sigma_{X}\right.$, $\sigma_{Y}, \sigma_{Z}, \tau_{X Y}, \tau_{Y Z}$ e $\tau_{Z X}$, ao longo do comprimento de cada fio modelado. Para tanto, foram exportados e analisados os valores das componentes de tensão em todos os nós da linha de centro do filamento. O padrão de tensão residual identificado por este procedimento foi aplicado no modelo de uma peça fabricada em ABS com geometria definida conforme Cattenone et al. (2019). Os valores de deslocamento obtidos pelo modelo foram comparados com os resultados experimentais nos pontos de medição ilustrados na Figura 3, conforme apresentados pelos autores. 


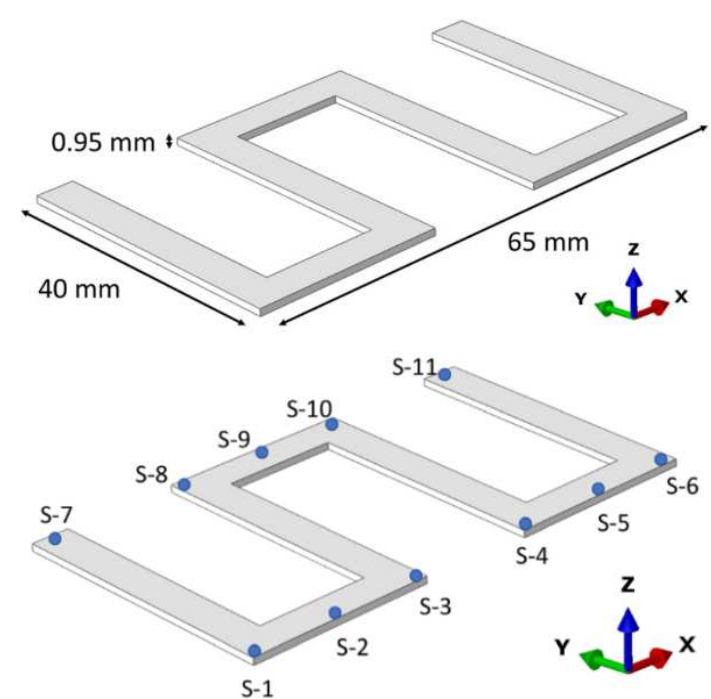

Figure 3 - Geometria da peça e pontos de medição experimentais de deslocamento. Fonte: Cattenone et al.

(2019).

\section{RESULTADOS E DISCUSSÃO}

A Figura 4 ilustra a evolução do campo de temperatura obtida pela análise térmica transiente no fio de $15 \mathrm{~mm}$ de comprimento para diferentes tempos de simulação, representando a translação do bico extrusor.

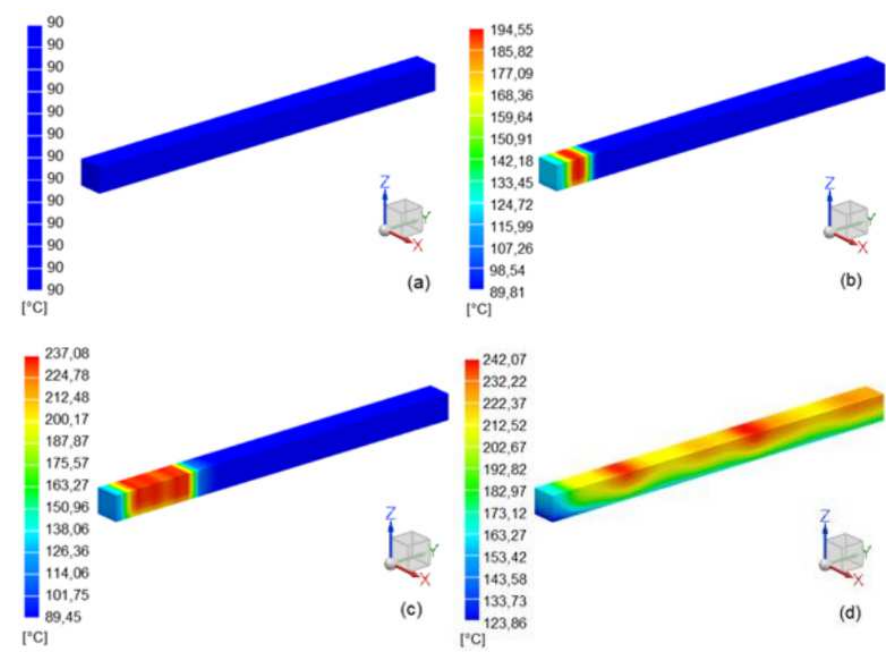

Figura 4 - Temperaturas do filamento de $15 \mathrm{~mm}$ antes de iniciar a impressão (a), no início da impressão (b), após 0,2 s de impressão (c) e ao término da impressão (d).

Pode-se observar que no início da simulação todo o filamento está na mesma temperatura da mesa de $90^{\circ} \mathrm{C}$ e então ocorre o aquecimento gradual da porção corresponde a posição do bico extrusor. O comportamento dos outros quatro fios de comprimentos diferentes $(20 \mathrm{~mm}, 40 \mathrm{~mm}, 80 \mathrm{~mm}$ e $160 \mathrm{~mm})$ simulados é análogo ao da Figura 4 . O padrão das componentes de tensão atuantes, normais e de cisalhamento $\left(\sigma_{X}, \sigma_{Y}, \sigma_{Z}, \tau_{X Y}\right.$, $\tau_{\mathrm{YZ}}$ e $\left.\tau_{\mathrm{ZX}}\right)$, ao longo do comprimento dos fios é mostrado na Figura 5. As componentes de cisalhamento $\tau_{X Y}$ e $\tau_{Z X}$ foram nulas em todo o comprimento dos seis fios. O eixo das abscissas dos gráficos varia de 0 a $\mathrm{L}$ e representa o comprimento dos filamentos, dessa forma é possível comparar o padrão das tensões em todos os fios de forma normalizada.
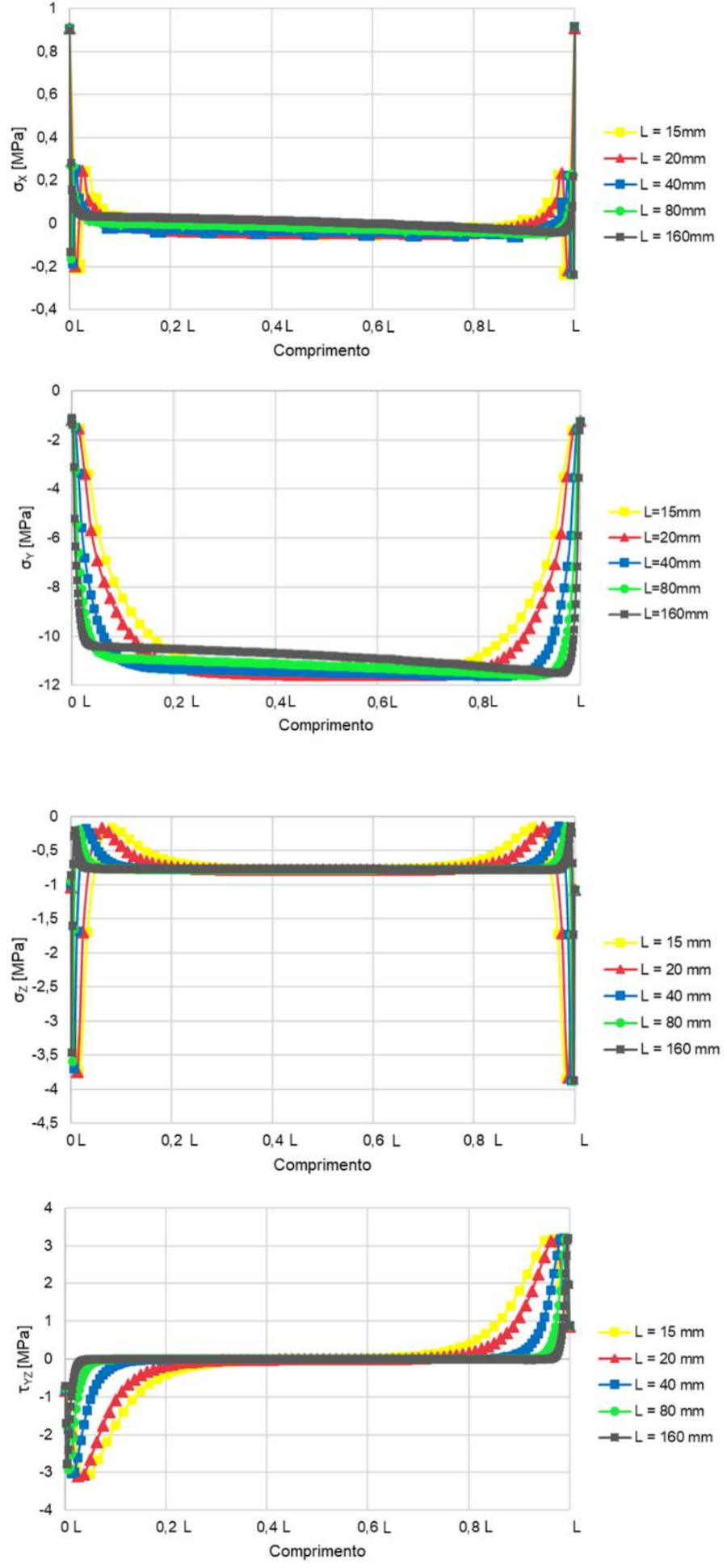

Figure 5 - Padrão das componentes de tensão ao longo do comprimento dos fios.

Analisando cada uma das componentes, percebe-se que a tensão normal $\sigma_{\mathrm{X}}$ e a cisalhante $\tau_{\mathrm{YZ}}$ possuem valores nulos ao longo de praticamente todo o comprimento dos filamentos, apresentando um valor considerável apenas nas extremidades. Para a tensão normal $\sigma_{\mathrm{Z}}$ o comportamento é semelhante, apresentado um baixo valor de tensão (aproximadamente -0,75 $\mathrm{MPa}$ ) para todo o comprimento dos fios e um valor maior nas extremidades. No entanto, a tensão axial $\sigma_{Y}$ possui tensão compressiva de aproximadamente $11 \mathrm{MPa}$ ao longo do comprimento dos fios, possuindo grande influência na deformação da peça, devido à sua magnitude elevada. Analisando os diferentes comprimentos de filamentos 
depositados, pode-se verificar o mesmo comportamento de tensões residuais, inclusive em magnitude, evidenciando que, para as hipóteses assumidas no procedimento, o comprimento do fio é um fator que pode ser desconsiderado quando se está avaliando o comportamento das tensões residuais.

O padrão das tensões residuais identificado foi aplicado no modelo ilustrado na Figura 6, correspondente a geometria e propriedades da peça analisada no trabalho de Cattenone et al. (2019). Neste modelo, considerou-se como condições de contorno o travamento dos nós nas regiões que experimentalmente os autores constataram que permaneceu aderida à mesa.

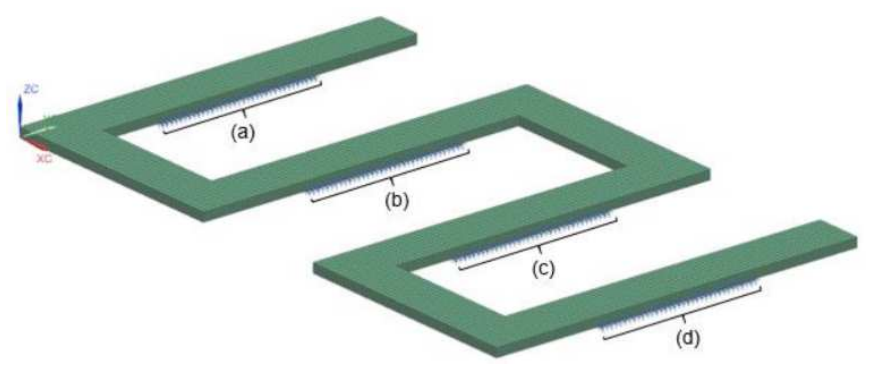

Figura 6 - Modelo de elementos finitos com identificação das regiões com aplicação de condições de contorno.

A distorção final obtida na peça pelo modelo de elementos finitos, correspondente ao seu deslocamento vertical, é ilustrado na Figura 7. Pode-se observar que o padrão de distorção obtido pelo modelo apresenta boa correlação com o padrão ilustrado na foto da peça (Figura 8) captada após sua impressão no trabalho de Cattenone et al. (2019).

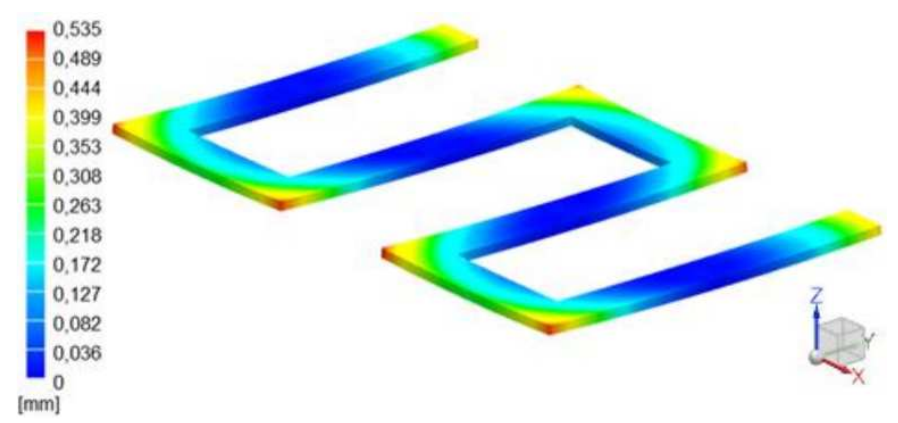

Figura 7 - Distorção obtida pelo modelo de elementos finitos.

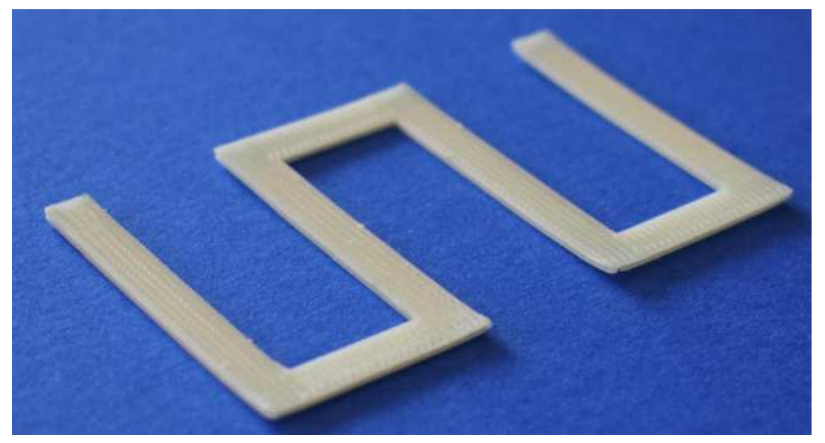

Figura 8 - Foto da peça após sua impressão. Fonte: Cattenone et al. (2019).

A Tabela 1 apresenta a comparação do erro relativo entre os deslocamentos obtidos pelo modelo proposto $\left(\mathrm{u}_{\mathrm{z}}{ }^{\mathrm{m}}\right)$ e os valores de deslocamentos experimentais $\left(\mathrm{u}_{\mathrm{z}}^{\mathrm{e}}\right)$ dos pontos de medição utilizados por Cattenone et al. (2019).
Tabela 1 - Comparação entre os deslocamentos do modelo proposto e experimentais.

\begin{tabular}{cccc}
$\begin{array}{c}\text { Ponto de } \\
\text { medição }\end{array}$ & $\begin{array}{c}\text { Deslocamentos } \\
\text { do modelo } \\
\mathbf{u}_{\mathbf{z}}{ }^{\mathbf{m}}[\boldsymbol{\mu m}]\end{array}$ & $\begin{array}{c}\text { Deslocamentos } \\
\text { experimentais } \\
\mathbf{u}_{\mathbf{z}}{ }^{\mathbf{e}}[\boldsymbol{\mu m}]\end{array}$ & $\begin{array}{c}\text { Erro relativo } \\
{[\%]}\end{array}$ \\
\hline S1 & 475 & 376 & 21 \\
S2 & 250 & 269 & 8 \\
S3 & 468 & 382 & 18 \\
S4 & 484 & 348 & 28 \\
S5 & 251 & 268 & 7 \\
S6 & 465 & 372 & 20 \\
S7 & 378 & 296 & 22 \\
S8 & 436 & 412 & 6 \\
S9 & 237 & 256 & 8 \\
S10 & 463 & 422 & 9 \\
S11 & 404 & 508 & 26 \\
\hline
\end{tabular}

\section{CONCLUSÕES}

O procedimento desenvolvido para a simulação do processo de manufatura aditiva FDM para previsão da distorção de componentes se mostrou uma alternativa mais simples e rápida em relação a abordagem que emprega o recurso de ativação e desativação dos elementos.

A abordagem proposta permitiu avaliar o padrão das tensões residuais atuantes em um filamento polimérico quando este é depositado. Identificou-se que a tensão axial é a componente que possui maior magnitude e por isso tem grande influência nos deslocamentos finais da peça produzida.

A modelagem proposta para prever a distorção final de uma peça em ABS produzida pelo método FDM atingiu bons resultados, apesar das simplificações adotadas. Os erros entre o deslocamento vertical obtido experimentalmente por Cattenone et al. (2019) e os obtidos nesse estudo encontram-se entre $6 \%$ e $28 \%$.

\section{AGRADECIMENTOS}

Os autores gostariam de agradecer ao Departamento de Engenharia Mecânica (DEMec) da Universidade Federal de São Carlos (UFSCar) pela motivação e infraestrutura fornecida.

\section{R E F E R E N C E S}

ARMillotTA, A.; BELlotTI, M.; CAVAllaRO, M. Warpage of FDM parts: Experimental tests and analytic model. Robotics and Computer-Integrated Manufacturing, v. 50, p. 140-152, 2018.

CATTENONE, A.; MORGANTI, S.; ALAIMO, G.; AURICCHIO, F. Finite element analysis of additive manufacturing based on fused deposition modeling: distortions prediction and comparison with experimental data. Journal of Manufacturing Science and Engineering, v. 141, n. 1, p. 1-17, 2019.

EL MOUMEN, A.; TARFAOUI, M.; LAFDI, K. Modelling of the temperature and residual stress fields during 3D printing of polymer composites. The International Journal of Advanced Manufacturing Technology, v. 104, p. 1661-1676, 2019. 
GIBSON, I; ROSEN, D.; STUCKER, B. Additive Manufacturing Technologies: 3D Printing, Rapid Prototyping and Direct Digital Manufacturing. $2^{\text {nd }}$ ed. New York: Springer, 2015.

LI, H.; WANG, T.; LI, Q.; YU, Z.; WANG, N. A quantitative investigation of distortion of polylactic acid/PLA) part in FDM from the point of interface residual stress. The International Journal of Advanced Manufacturing Technology, v. 94, p. 381-395, 2018.

YANG, H.; ZHANG, S. Numerical simulation of temperature field and stress field in fused deposition modeling. Journal of Mechanical Science and Technology, v. 32, n. 7, p. 3337-3344, 2018.
ZHANG, Y.; CHOU, Y. K. Three-dimensional finite element analysis simulations of the fused deposition modelling process. In: PROCEEDINGS OF THE INSTITUTION OF MECHANICAL ENGINEERS, Part B: Journal of Engineering Manufacture, v. 220, n. 10, p. 1663-1671, 2006.

ZHANG, Y.; CHOU, Y. K. A parametric study of part distortions in fused deposition modelling using threedimensional finite element analysis. In: PROCEEDINGS OF THE INSTITUTION OF MECHANICAL ENGINEERS, Part B: Journal of Engineering Manufacture, v. 222, n. 8, p. 959-968, 2008. 